\title{
Practical Applications of the Mathematical Representation for Turbulent Recirculatory Flows*
}

\section{By Julian SZEKELY ${ }^{* *}$ and Shigeo ASAI***}

\section{Synopsis}

In the paper practical applications are presented of the mathematical representation developed in a previous paper ${ }^{1)}$ for turbulent recirculatory flows.

The examples discussed in the paper include mixing in argon stirred ladles, the flow patterns in the liquid pool of continuous casting systems, the flow field induced by an electromagnetic force field in continuous casting and flow patterns in rimming ingots.

The computed results indicate velocities as high as $30 \mathrm{~cm} / \mathrm{sec}$ may be found in these systems and that the eddy diffusivities may have values as high as $500 \mathrm{~cm}^{2} / \mathrm{sec}$. The computed results were found to be in reasonable semi-quantitative and in some cases, quantitative agreement with the results of experimental model tests. While the computer requirements are quite substantial, some 5 to $10 \mathrm{~min}$ on a DCD 6400, the approach outlined here seems attractive because it provides a much improved insight into the structure of the flow field and a more solid basis for further studies of the coalescense and entrapment of inclusions, electromagnetic stirring, and mass transfer in steel processing.

\section{Introduction}

In the previous paper $^{1)}$ a general mathematical description of two-dimensional or axi-symmetrical turbulent recirculatory flows was presented together with the computational algorithms that are available for the solution of the resultant system of differential equations. It has to be stressed that at present our understanding of turbulence phenomena is rather incomplete and that even the use of quite crude turbulence models, such as used in the paper, require substantial amounts of computer time. However, this new approach to fluid flow problems in steel processing is thought to be worthwhile because of its potential for providing a much improved insight into the detailed transport mechanism in these systems.

In the following we shall illustrate the application of the previously presented analysis for representing several practical steel processing systems, stressing wherever possible, the comparison between the predictions based on the model with actual experimental measurements. Such direct comparison is thought to be essential for the verification of these models for steel processing conditions and for the eventual refinement of these ideas.

The following particular systems will be considered:

1) Mixing in argon-stirred ladles

2) Fluid flow and its effects in continuous casting

3) Fluid flow field induced by electromagnetic stirring

4) Flow pattern in rimming ingots.

\section{Applications of the Model}

In the following, we shall illustrate how the model may be used for calculating the velocity field and the mixing characteristics in a number of basic physical situations of importance in steel processing.

\section{Mixing in Argon-stirred Ladles ${ }^{2}$}

Problems of this type are of considerable practical importance because such stirring arrangements are frequently used for the homogenization of heats and also for promoting the flotation of inclusions.

Let us consider a cylindrical ladle containing molten steel which is agitated by the passage of a gas stream through a porous plug located in the center line at the bottom, as sketched in Fig. 1.

The problem may be stated by writing Eqs. (1) to (6), (8) to (10), (14) and (15) given in the previous paper $^{1)}$ and the appropriate expressionsfor the turbulent viscosity together with the boundary conditions. In this case the "Two Equation Model" was used for the turbulent viscosity and the boundary conditions had to express the following:

(1) The stream function must be constant at the free surface and at the walls because no normal velocity components may exist at these locations. The

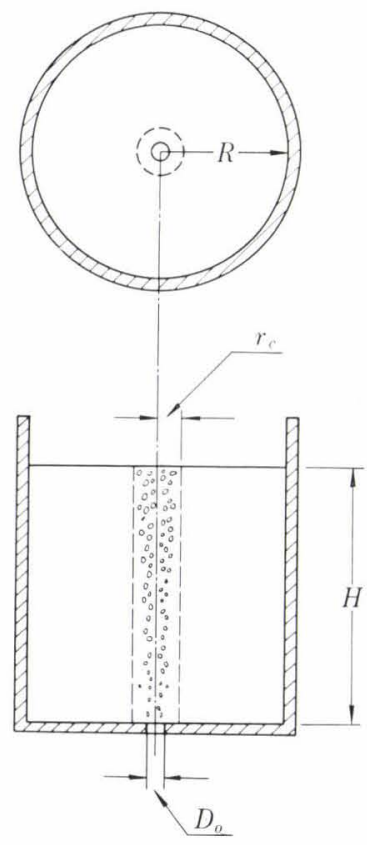

Fig. 1. Sketch of ladle stirred by argon gas

* Received July 17, 1974.

** Department of Chemical Engineering and Center for Process Metallurgy, State University of New York at Buffalo, Buffalo, N.Y. 14214, USA.

*** Department of Chemical Engineering and Center for Process Metallurgy State, University of New York at Buffalo. On leave from Department of Iron and Steel Engineering, Nagoya University, Chikusa-ku, Nagoya 464. 
stream function at the surface of the central core may be obtained from a momentum balance.

(2) The vorticity is zero at the free surface and its value at the wall may be deduced from the no slip condition between the wall and the adjacent fluid; this mathematical expression was proposed by Gosman, et $a l^{3)}$ The value of vorticity at the surface of the central core may be obtained from a relationship between the stream function and the local shear stress.

(3) The normal gradients of the turbulent energy and of the characteristic are zero at the free surface. The turbulent energy and the characteristic $(W)$ at the wall and at the surface of the central core may be related to the local shear stress.

\section{Results}

In order to test the mathematical model (re-worked for a two dimensional system) physical modeling experiments were carried out in a system where water contained in an essentially two-dimensional rectangular slot $(47 \mathrm{~cm} \times 47 \mathrm{~cm} \times 2.5 \mathrm{~cm})$ was agitated by gas bubbles introduced through an orifice located at the bottom. The actual measurements taken included the establishment of the fluid flow pattern through the use of polystyrene beads as tracers and time lapse photography and the determination of the mixing time by using dye tracer additions. Photograph 1 shows the experimentally determined flow field which indicates that the injected gas stream at the center produces two symmetrically located roll cells. Photograph 2 shows good agreement between measurements and predic-

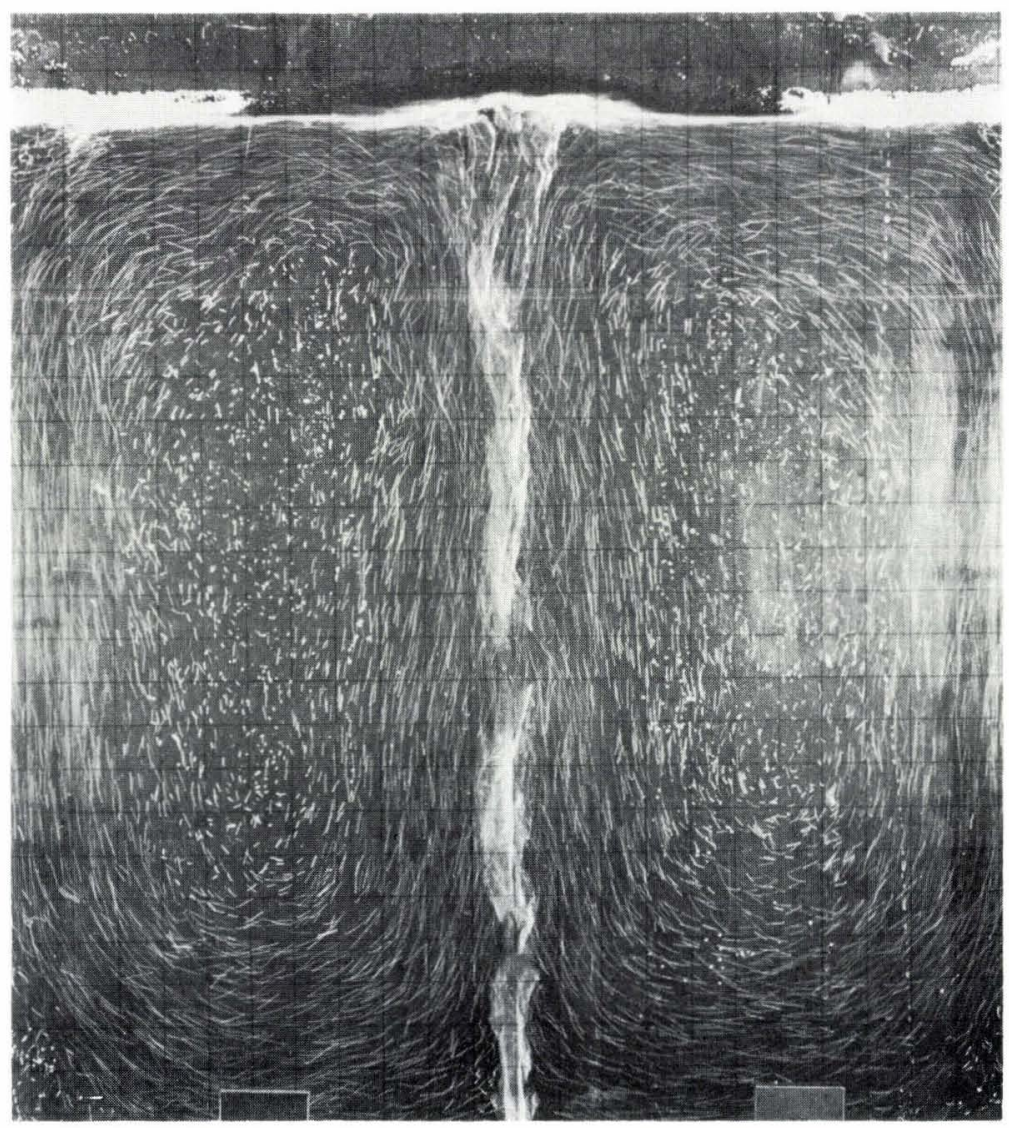

Photo. 1. Flow pattern in the two-dimensional model experiment tions, with the computed streamline pattern being given on the right hand side of the plot.

The computed velocity distribution is given in Fig. 2 where both the direction and the absolute magnitude of the velocity vector are indicated. It is noted that the model predicts unrealistically high velocities at the free surface in the vicinity of the center - which is probably attributable to the oversimplified representation of the free surface. The remainder of the velocity field, however, seems realistic and consistent with ac-

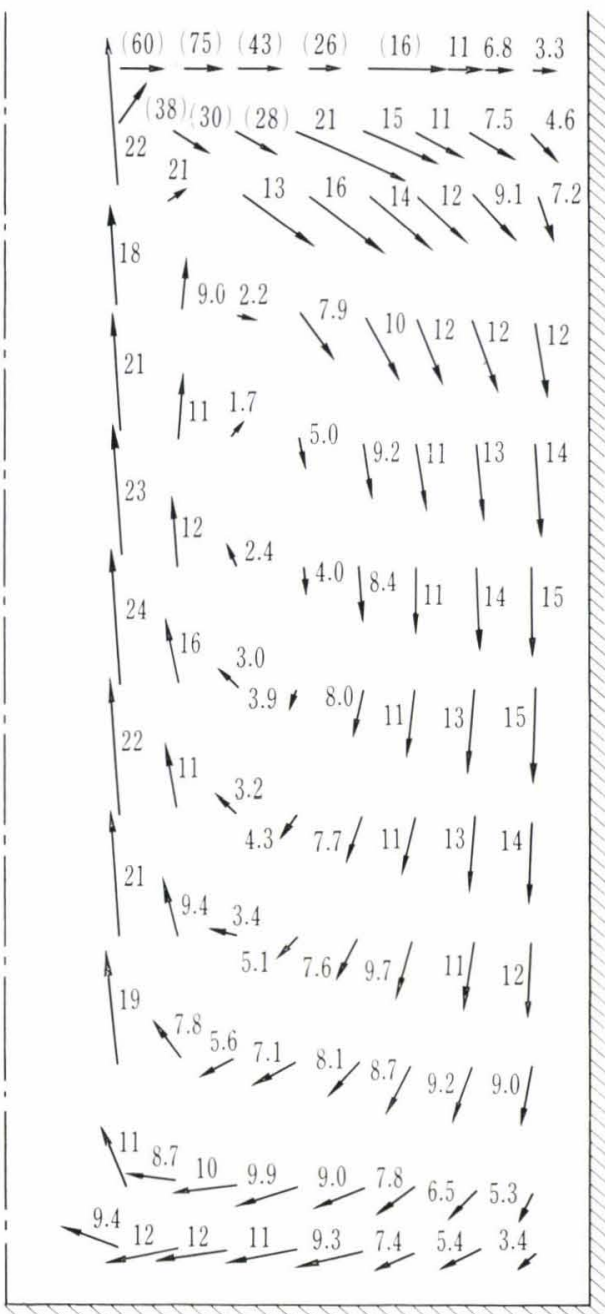

Fig. 2. Computed velocity profiles (arrows denote thedirection of the velocity vector and the numbers correspond to the absolute values of velocity, $\mathrm{cm} / \mathrm{sec}$

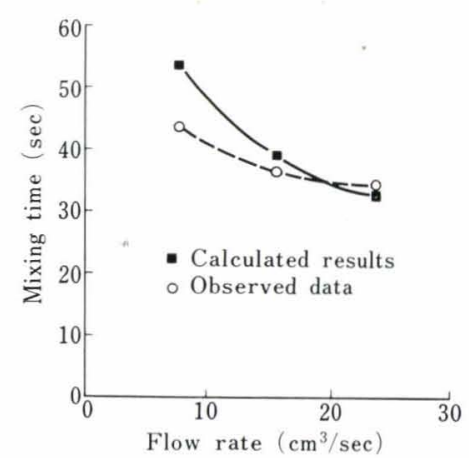

Fig. 3.

Comparison of the predicted and the experimentally measured mixing times for the water model tests 


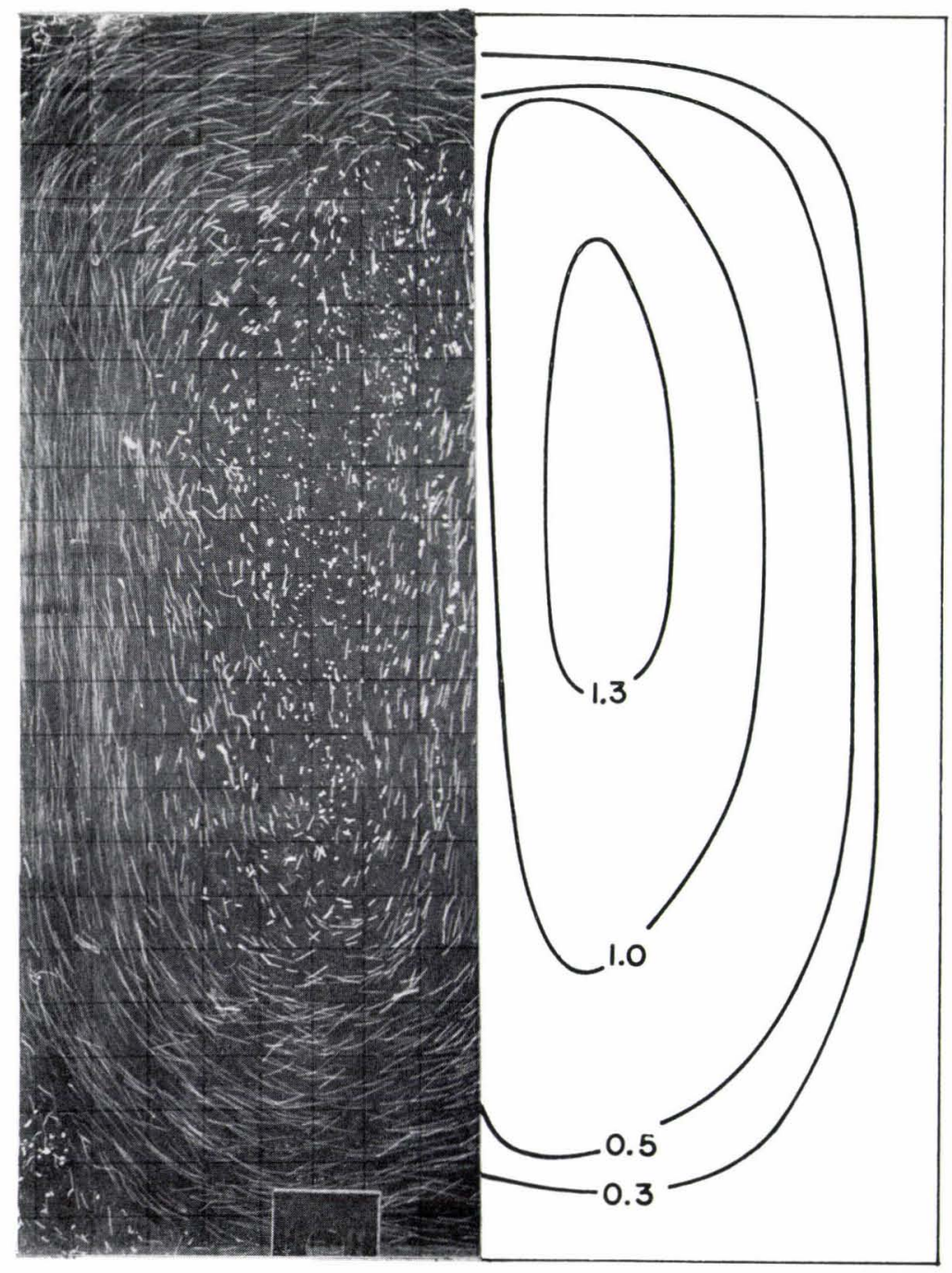

Photo. 2. Comparison of the computed and the measured streamline patterns (numbers on curves denote values of dimensionless stream function)

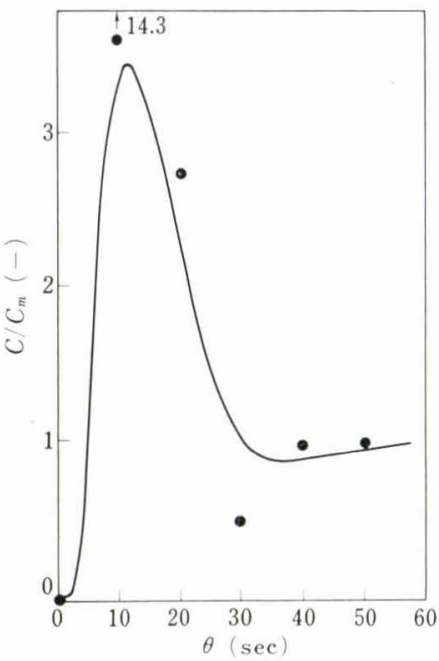

Fig. 4

Comparison of experimentally measured and the predicted concentration profiles in a VOD system

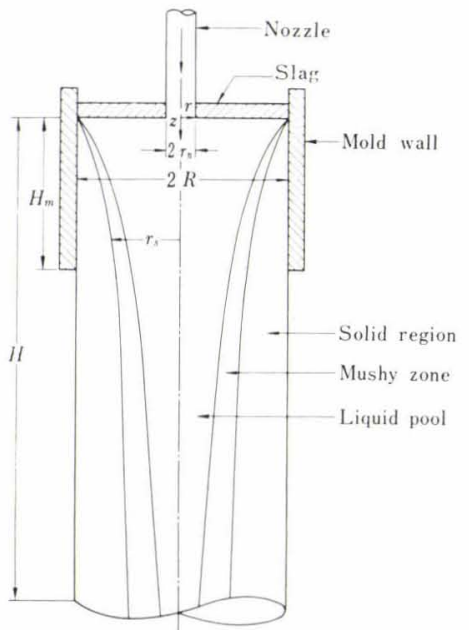

Fig. 5. A sketch of a continuous casting system tual visual observations, viz high velocities near the wall and a relatively quiescent region in the center, readily observable on Photos. 1 and 2.

Figure 3 shows a plot of the experimentally determined mixing time against the air flow rate for the model experiments. Also shown, with full squares are the computed values of the mixing time; the fairly good agreement between measurements and predictions is readily apparent.

Ultimately our objective in this work is to predict the behavior of real metallurgical systems. A recent paper by Nakanishi, et al. ${ }^{4)}$ provides an excellent example of a careful tracer study aimed at the determination of flow patterns; in this work the authors introduced radioactive cobalt 60 into a $50 \mathrm{t}$ VOD furnace and then determined the radioactivity of the samples taken at $10 \mathrm{sec}$ intervals.

Figure 4 shows a comparison between the experimental measurements and the predictions based on the model; since the experimental system did not exhibit axial symmetry, while such symmetry had to be as- sumed in the calculations, the apparently good agreement between measurements and predictions should be regarded as semi-quantitative only. Nonetheless, even this semi-quantitative agreement should be quite encouraging because the computation was based on fluid flow fundamentals rather than on empirical data fitting.*

\section{Fluid Flow and Its Effects in Continuous Casting}

The widespread interest in continuous casting has stimulated a great deal of research effort into various aspects of this process. The earlier experimental and theoretical studies aimed at predicting the thickness of the solidified shell were followed by more recent investigations which were concerned with the factors that govern surface quality and the inclusion count in the solidified product. ${ }^{5-8)}$ In this regard useful water modeling studies have been reported by numerous investigators ${ }^{9-12)}$ which were augmented by both theoretical analyses and by tracer dispersion measurements.

As a result of this work, a reasonably good qualita-

* A more detailed study of mixing in a VOD furnace will be described in a forthcoming publication. 
tive picture is now available of the continuous casting process although our understanding of these phenomena is far from complete.

It is to be noted that all the thermal analyses that have been made neglected the fluid flow effects, and turbulent mixing in the mold region was represented by assuming an artificially high "effective" value for the thermal conductivity of the molten steel. Moreover, in the analyses aimed at the quantitative description of the fluid flow field, the effect of heat transfer and solidification were not taken into consideration so that these results too were somewhat overstimplified.

In the calculations to be presented here a more sophisticated mathematical model is given of the continuous casting process where account is taken of both heat transfer (solidification, the development of the mushy zone, etc.) and of the turbulent fluid flow of the melt within the pool region. Such an approach, while posing a more complex computational task, will tend to provide a much more accurate representation of continuous casting systems; for this reason an attempt will be made to compare the predictions based on the model with actual plant scale measurements regarding the solidification profiles and tracer dispersion. Furthermore, the model may also be used for predicting the entrapment pattern of non-metallic inclusions for various operating conditions.

Let us consider the upper portion of a continuous casting system where the liquid pool is agitated by the metal stream poured from a nozzle, as sketched in Fig. 5. The mathematical statement of the fluid flow problem is given in Eqs. (1) to (11) in the previous paper ${ }^{1}$ together with the expression for the turbulent viscosity and the boundary conditions. For this particular case the One Equation Model was used for representing the turbulent viscosity and Nikuradse's expression was used for estimating the mixing length.

In writing the thermal energy balance equation, rather than using Eq. (18) in the previous paper ${ }^{1)}$ and developing separate relationships for the two-phase (mushy) zone and the moving solidification front, the following equations were used, which is valid for the melt, the mushy zone and the completely solidified shell:

$$
\left\{1+\left(\frac{(-\Delta H)}{C_{p}} \frac{\partial f_{l}}{\partial T}\right)\right\}\left\{\frac{\partial}{\partial Z}\left(T \frac{\partial \psi}{\partial r}\right)-\frac{\partial}{\partial r}\left(T \frac{\partial \psi}{\partial Z}\right)\right\}
$$

(heat generation in the (convective term) mushy zone)

$$
\begin{aligned}
& =\rho \frac{\partial}{\partial r}\left\{r\left(k+\frac{k_{t}}{\sigma_{T}}\right) \frac{\partial T}{\partial r}\right\}+\rho \frac{\partial}{\partial Z}\left\{r\left(k+\frac{k_{t}}{\sigma_{T}}\right) \frac{\partial T}{\partial Z}\right\} \quad \ldots(1)^{*} \\
& \text { (heat transfer by eddy and by molecular conduction) }
\end{aligned}
$$

where, $f_{l}$, the liquid fraction appearing in Eq. (1) may be estimated by assuming equilibrium freezing:

$$
f_{\imath}=1-\frac{1}{m-1}\left\{\frac{M C_{i}}{T_{m}-T}-1\right\}=1-f_{s}
$$

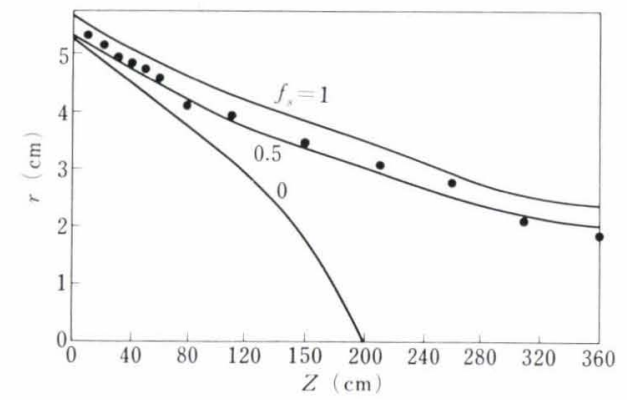

Fig. 6. Comparison of the computed profiles of solid fraction with the experimental measurements of the shell thickness obtained by Ushijima ${ }^{14)}$

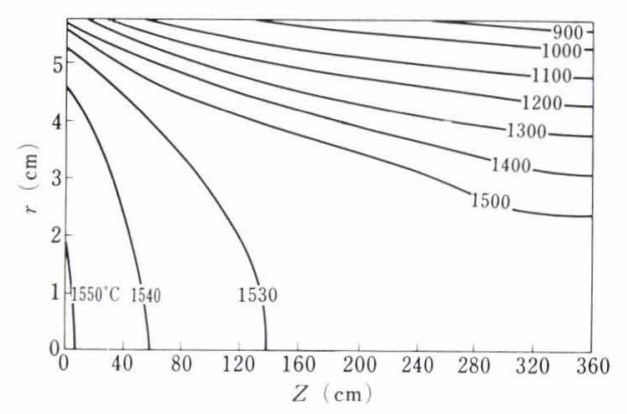

Fig. 7. Computed temperature profiles for conditions

\begin{tabular}{|c|c|}
\hline Diameter of mold & $11.5 \mathrm{~cm}$ \\
\hline Diameter of nozzle* & $3 \mathrm{~cm}$ \\
\hline Mold length* & $50 \mathrm{~cm}$ \\
\hline Casting speed & $3.17 \mathrm{~cm} / \mathrm{sec}$ \\
\hline Casting temperature & $1550^{\circ} \mathrm{C}$ \\
\hline Carbon content & $0.11 \%$ \\
\hline Equilibrium partition coefficient & $0.2(-)$ \\
\hline $\begin{array}{l}\text { Heat transfer coefficient in } \\
\text { the mold** }\end{array}$ & $0.0306 \mathrm{cal} / \mathrm{cm}^{2} \mathrm{sec}$ \\
\hline $\begin{array}{l}\text { Heat transfer coefficient below } \\
\text { the mold** }\end{array}$ & $0.0258 \mathrm{cal} / \mathrm{cm}^{2} \mathrm{sec}^{\circ}$ \\
\hline Thermal conductivity of steel & $0.827 \mathrm{cal} / \mathrm{cm} \mathrm{sec}^{\circ} \mathrm{C}$ \\
\hline \multicolumn{2}{|c|}{$\begin{array}{l}\text { These were guessed. } \\
\text { These were estimated from Ref. 19). }\end{array}$} \\
\hline $\begin{array}{ll}\text { if } f_{l} \geq 1 & f_{l}=1 \\
\text { if } 0<f_{l}<1 & f_{l}=f_{l} \\
\text { if } f_{l} \leq 0 & f_{l}=0\end{array}$ & $\begin{array}{l}\text { liquid zone } \\
\text { mushy zone } \\
\text { solid zone }\end{array}$ \\
\hline
\end{tabular}
specified in Table 1

Table 1. Numerical values of the parameters used in the calculation of Figs. 6 to 9

where, $T_{m}$ : melting point of the solvent

$M:$ slope of a liquidus line

$m$ : equilibrium partition ratio

$C_{i}$ : initial concentration of solute

$f_{s}$ : solidified fraction.

Equation (1) is valid for the melt, the mushy and solid regions because the derivative of the liquid fraction, $\left(\partial f_{l} / \partial T\right)$ is zero by definition in both the liquid and the solid regions. A selected set of the computed

* The procedure for representing the solid, mushy and molten regions in terms of a single equation and allowing for the latent heat liberated through an " effective temperature dependent specific heat" has been used extensively in the modeling of continuous casting systems. The present method is, however, an extension of these previous techniques because here account was taken of convective effects. 


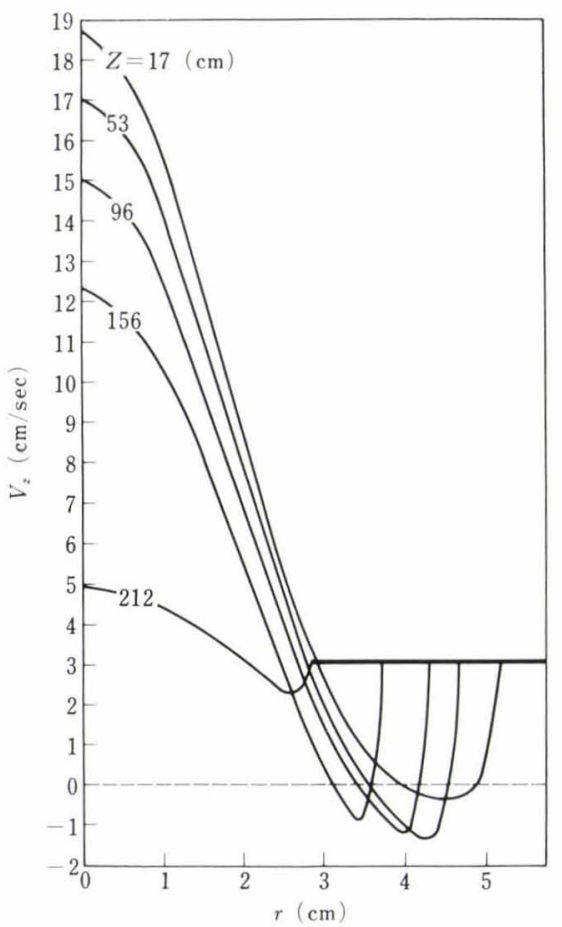

Fig. 8. Computed axial velocity profiles at various axail positions $(Z)$ for the conditions given in Table 1

results is shown in the following Figs. 6 to 11. A more extensive treatment of this problem will also be available in a forthcoming publication. ${ }^{13)}$

Figures 6 to 9 show the behavior of a billet caster, the key operating parameters of which are given in Table 1. Figure 6 shows the computed profiles corresponding to solid fractions of $1,0.5$ and 0 , respectively; also shown, for the purpose of comparison, are the plant-scale measurements of Ushijima. ${ }^{14}$ It is seen that the experimentally measured shell thickness profile seems consistent with the profile corresponding to a solid fraction of 0.5 , which would indicate that only part of the mushy zone adheres to the solidified shell.

Figure 7 shows the computed temperature profiles where it is seen that superheat is removed very rapidly from the liquid pool; this is consistent with earlier observations. However, in contrast to earlier mathematical modeling work, ${ }^{15}$ ) where the rapid removal of the superheat had to be represented by arbitrarily increased "effective conductivity" the turbulent flow model allows the description of this phenomenon in terms of more fundamental considerations.

Figure 8 shows a plot of the profiles of $v_{z}$, the vertical velocity component, with the vertical distance from the pouring nozzle as a parameter. Also shown, with the heavy horizontal line, is the vertical velocity corresponding to the casting speed. The completely solidified region will be moving with this velocity throughout. Inspection of Fig. 8 also indicates that while the region of reverse flow would be confined to a relatively small region, if we were to consider a fixed frame of reference, the reverse flow region would become quite substantial if we consider velocities relative to the casting speed. This latter is, of course, the proper frame of reference.

Figure 9 shows the entrapment pattern of non-

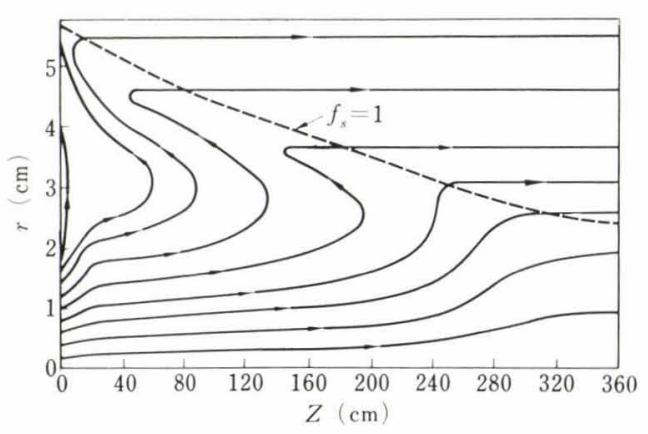

Fig. 9. Computed location of the non-metallic inclusions for $100 \mu$ particles for the conditions specified in Table 1. The broken line indicates the curve corresponding to 1.0 solid fraction

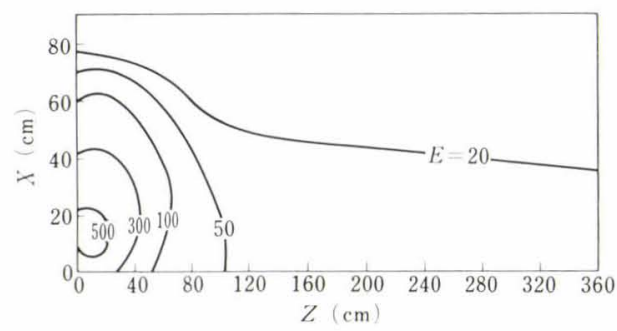

Fig. 10. Computed values of the effective diffusivity in $\mathrm{cm}^{2} / \mathrm{sec}$

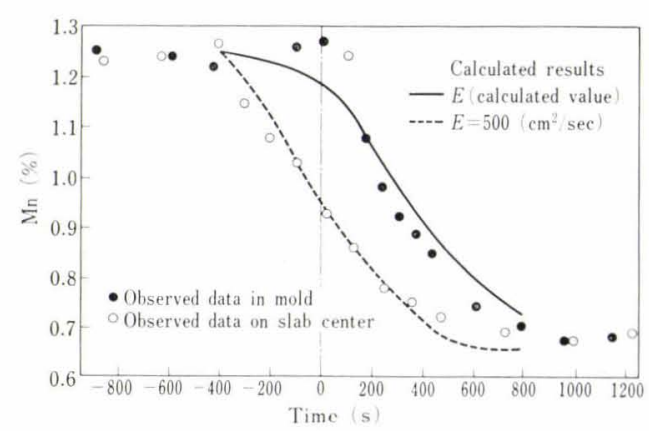

Fig. 11. Comparison of the computed concentration profiles of $\mathrm{Mn}$ with the experimental data by Fujii ${ }^{16)}$

Table 2. Numerical values of the parameters used in the calculation of Figs. 10 and 11

$\begin{array}{ll}\text { Size of mold } & 20 \times 170 \mathrm{~cm} \\ \text { Diameter of nozzle } & 6 \mathrm{~cm} \\ \text { Mold length* } & 50 \mathrm{~cm} \\ \text { Casting speed } & 1.2 \mathrm{~cm} / \mathrm{sec} \\ \text { Casting temperature } & 1550^{\circ} \mathrm{C} \\ \text { Carbon content } & 0.125 \% \\ \begin{array}{l}\text { Equilibrium partition coefficient } \\ \text { Heat transfer coefficient in } \\ \text { the mold** }\end{array} & 0.2(-) \\ \begin{array}{l}\text { Heat transfer coefficient below } \\ \text { the mold** }\end{array} & 0.0306 \mathrm{cal} / \mathrm{cm}^{2} \mathrm{sec}^{\circ} \mathrm{C} \\ \text { Thermal conductivity of steel } & 0.0258 \mathrm{cal} / \mathrm{cm}^{2} \mathrm{sec}^{\circ} \mathrm{C} \\ \text { This was guessed. } & 0.0827 \mathrm{cal} / \mathrm{cm} \mathrm{sec}^{\circ} \mathrm{C} \\ \text { These were estimated from Ref. } 19) .\end{array}$

metallic inclusions, $100 \mu$ in diameter, as calculated with the aid of the technique described in $I I .6$ of the previous paper. ${ }^{1)} \quad$ It is seen that for the casting speed and for the straight nozzle employed, most of the $100 \mu$ (and smaller particles) would have been entrapped. 

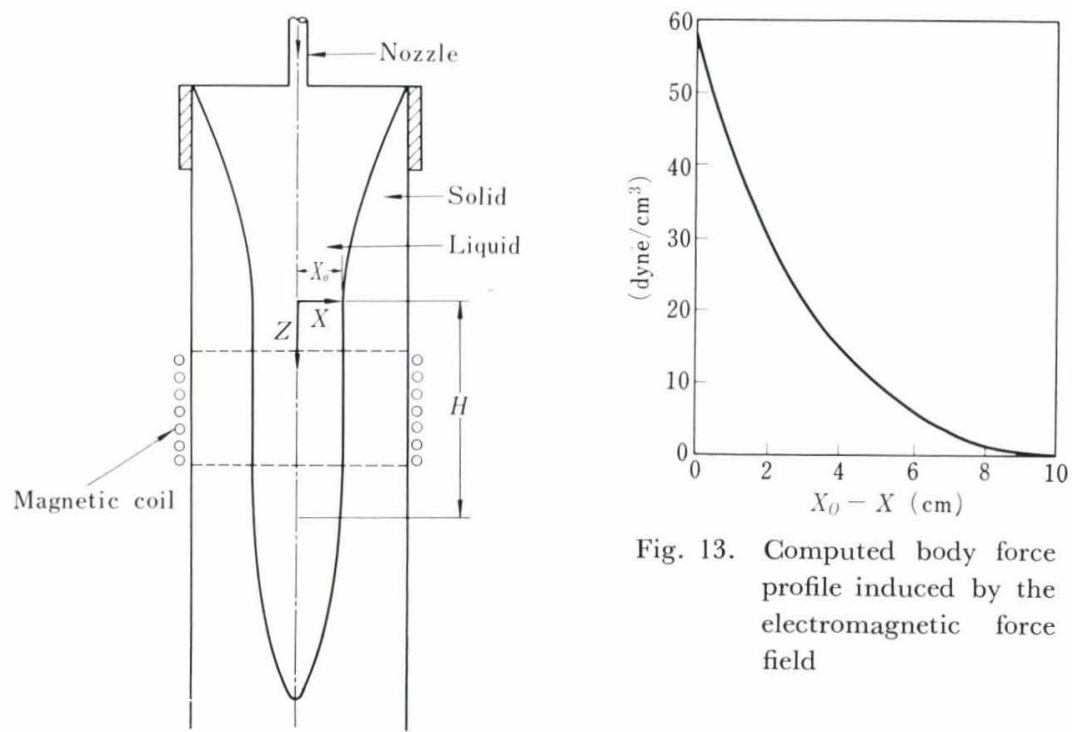

Fig. 13. Computed body force profile induced by the electromagnetic force field

Fig. 12. A sketch of a continuous slab caster with electromagnetic stirring

Table 3. Numerical values of the parameters used in the calculation of Figs. 13 to 18
Field size

Magnetic field length

Casting speed

Frequency

Magnetic field intensity at the melt-solid interface

Specific resistivity

Magnetic permeability

$20 \times 100 \mathrm{~cm}$
$50 \mathrm{~cm}$
$1 \mathrm{~cm} / \mathrm{sec}$
$30 \mathrm{~Hz}$
$470 \mathrm{G}$
$1.4 \times 10^{-6} \Omega \mathrm{m}$
$1.26 \times 10^{-6} \mathrm{H} \mathrm{m}^{-1}$

The technique outlined here could be used for the nozzle designs and continuous casting arrangements with a view of minimizing the entrapment of inclusions.

Finally, Figs. 10 and 11 show the behavior of a large slab casting machine, the principal operating parameters of which are listed in Table 2. The map of the computed eddy diffusivities is shown in Fig. 10; it is seen that the eddy diffusivity has very high values at the top of the mold (up to vertical distance of some 1 to 2 mold widths, measured from the meniscus) but the value of the eddy diffusivity is seen to decay quite rapidly for increasing values of $z$.

It is of interest to compare tracer dispersion, as predicted on the basis of the computed eddy diffusivities with that measured for a real continuous casting system (having the operating parameters listed in Table 2) as reported by Fujii..16) This is done in Fig. 11 where the data marked with the full circles refer to measurements made in the mold region, which thus constitute (in an approximate fashion) the input data for the dispersion model. The data designated by the empty circles correspond to samples taken from the center of the solidified slab and thus may be regarded as the response to the previously defined input.

The graph also shows two computed curves. The full line corresponds to the dispersion curve based on quantitative or at least semi-quantitative evaluation of

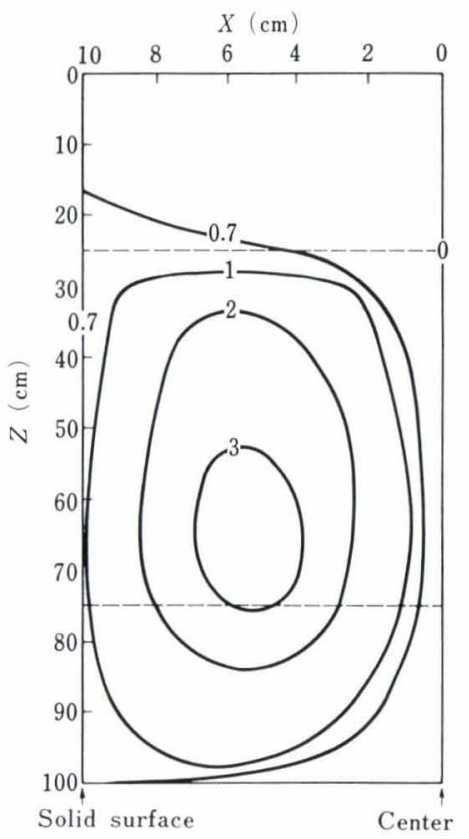

Fig. 14. Computed streamline pattern for the condition when the electromagnetic waves propagate in the upward direction the theoretically predicted (spatially variable) eddy diffusivity, the map of which was shown in Fig. 10. The broken line which appears to provide a much better representation for the experimental data, was calculated, somewhat arbitrarily, by assuming $E=500$ $\mathrm{cm}^{2} / \mathrm{sec}$, i.e., the largest theoretical value found for the system.

A partial explanation for the apparent discrepancy between measurements and prediction may be provided by the fact that the model is still oversimplified because no allowance has been made for the effect of the debris provided by the broken dendrites. Nonetheless, the model provides at least an approximate representation of dispersion phenomena in continuous casting.

In concluding the section dealing with continuous casting phenomena, it should be remarked that the combination of turbulent fluid flow analysis with a proper thermal representation of the solidification process suggested in this work should be very attractive potentially for both the interpretation of plant-scale dispersion measurements and ultimately for the design of optimal pouring nozzle configuration.

\section{Fluid Flow Field Induced by Electromagnetic Stirring}

It has been shown through both water model studies and through the computation of eddy diffusivities that in continuous casting intense mixing is confined to the upper mold region. It is understood furthermore, in part due to the pioneering work of Tsavaras, ${ }^{17)}$ a high shear field and steep velocity gradients would be desirable for hindering dendritic growth (more appropriately for promoting the shearing off of dendrite arms).

In order to restrict dendritic growth one may consider the application of an electromagnetic field with 


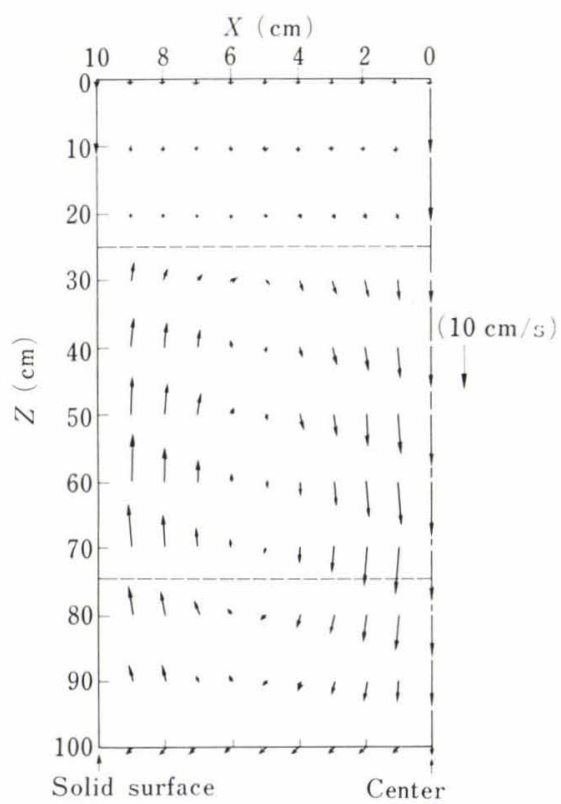

Fig. 15. Computed velocity profiles for the condition when the electromagnetic waves propagate in the upward direction

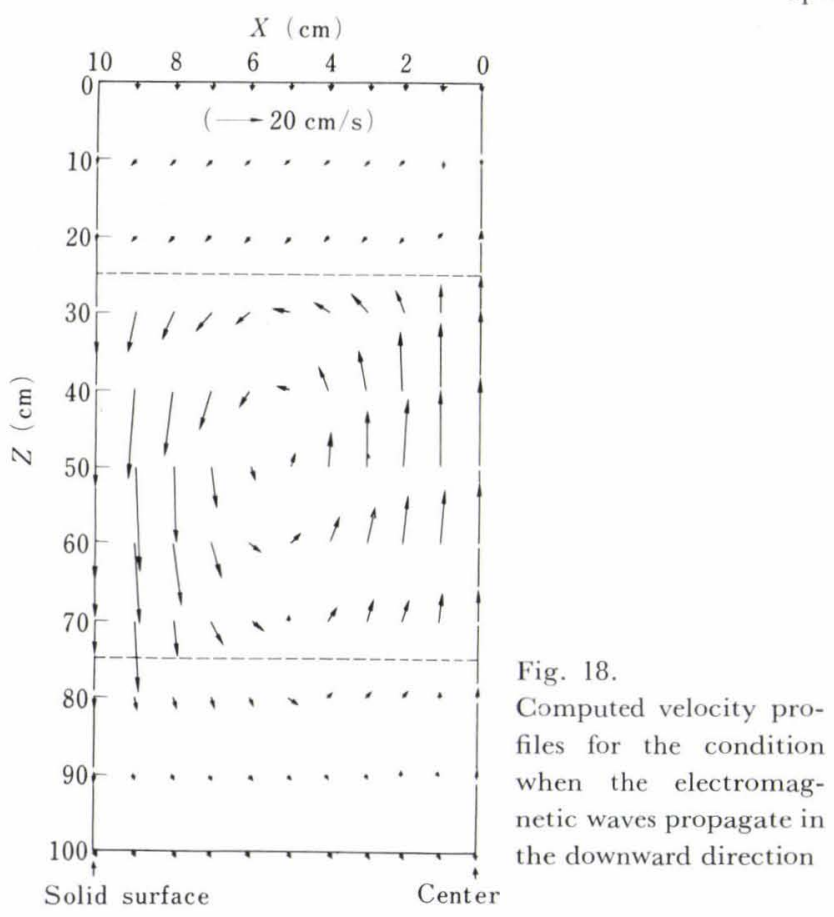

a view of promoting the circulation of the melt in the otherwise quiescent lower regions. Since such electromagnetically induced circulation is not readily measured, it would be desirable to devise methods for estimating the resultant flow fields. This may be done with the aid of the formulation developed in $I I .5$ of the previous paper. ${ }^{1)}$ Let us consider a continuous slab caster, such as sketched in Fig. 12, where the molten core some distance below the mold level is agitated by the application of an electromagnetic field. The principal operating parameters characterizing this system are listed in Table 3.

If we assume as first approximation that the solidi-

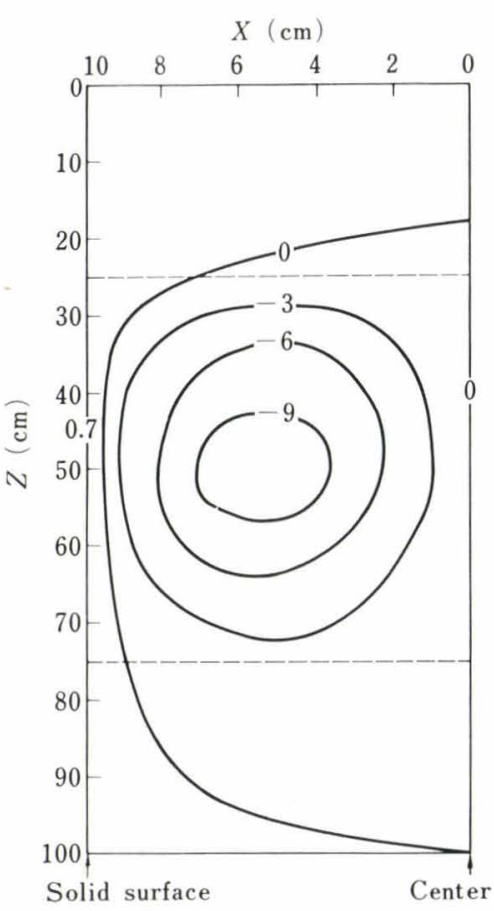

Fig. 17. Computed streamline pattern for the condition when the electromagnetic waves propagate in the downward direction

fication front may be represented by a vertical plane in the region where the electromagnetic field is applied, then the problem may be represented by Eqs. (1) to (10), (12) to (15) and (36) in the previous paper. ${ }^{1)}$ A selection of the computed results is given in the following Figs. 13 to 18.

Figure 13 shows the computed body force field induced by the electromagnetic force field for the conditions depicted in Table 3. It is readily seen that the body force decreases quite dramatically with distance from the solid-melt interface.

Figures 14 to 16 show computed results for the condition when the electromagnetic waves propagate in an upward direction, i.e., oppose the direction of casting.

Figure 14 shows the computed streamline pattern; it is noted that the region where the electromagnetic field is being applied is outlined by the two parallel horizontal broken lines. It is noted that the eye of the circulation is not located symmetrically, but rather, it is displaced in a downward direction. Figure 15 shows the velocity field corresponding to the streamline pattern depicted in Fig. 14. The velocity vector corresponding to a vertical velocity of $10 \mathrm{~cm} / \mathrm{sec}$ is also given, for the sake of comparison. Inspection of Fig. 15 indicates that the maximum upward velocity is of the order of $13 \mathrm{~cm} / \mathrm{sec}$ - in the vicinity of the solidification front, whereas the maximum downward velocity is of the order of $17 \mathrm{~cm} / \mathrm{sec}$ (both for the particular conditions considered) - in the vicinity of the center line.

Figure 16 shows the computed map of the turbulent energy; it is seen that quite high values of the turbulent energy may be induced by electromagnetic stirring. 


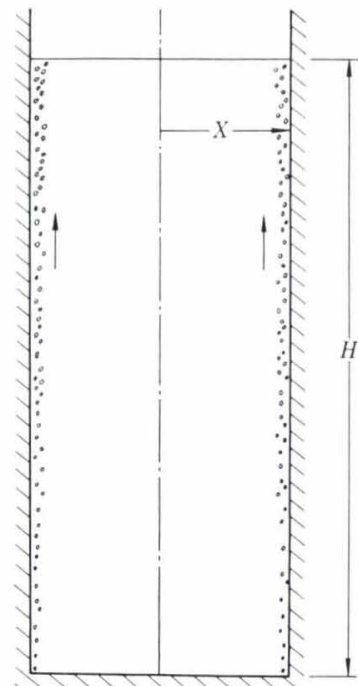

Fig. 19. Sketch of a rimming ingot

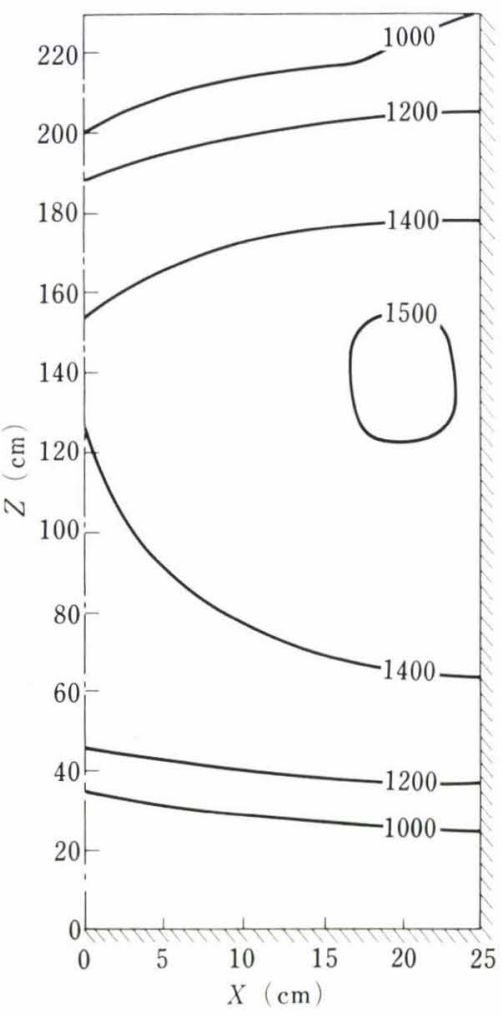

Fig. 22.

Computed turbulent energy profiles for a rimming ingot

Figures 17 and 18 depict a situation where the direction of wave propagation coincides with that of casting. It is seen that under such conditions the streamline pattern is nearly symmetrical, and that the intensity of circulation is much stronger. The computed velocity profiles are shown in Fig. 18 and it is seen that the fluid velocities are much higher than those given in the preceding Fig. 15 (a case where the propagation of the electromagnetic waves and the casting direction were opposed).

It is thought that through tackling the problem described in this section, entirely new ground has been broken because this is the first time that an albeit approximate description has been obtained of a turbulent flow field in continuous casting as induced by

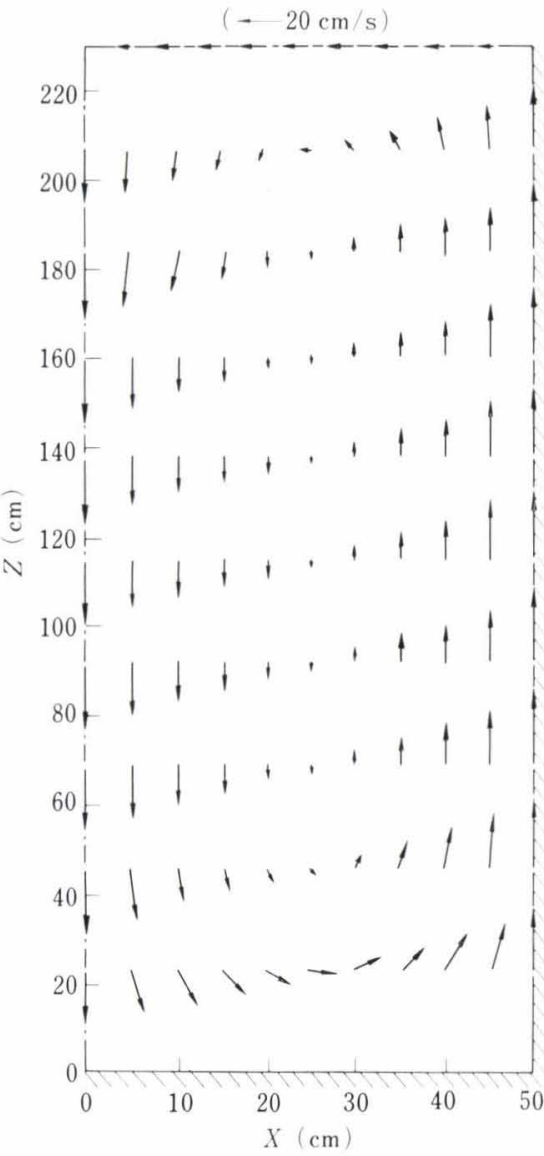

Fig. 21. Computed velocity profiles for a rimming ingot

electromagnetic forces. Further work that could be usefully undertaken in this area could involve the study of the interaction between the morphology of the solidified surface and the flow field induced by induction stirring. Another interesting area for future study would be to establish the optimum operating frequency for induction stirring as applied to continuous casting systems. Clearly, induction devices cannot work at a zero frequency; moreover, at very high frequencies all the induced fields and currents will be restricted to regions of the fluid where no motion can take place because of the non-slip boundary condition. It is very likely that there exists an optimum frequency between these two limiting cases; it is also probable that this optimum frequency would be a function of the geometry of the system.

\section{Flow Patterns in Rimming Ingots}

It is well-established that flow of the melt, caused by the " rimming action " may sweep away the solutes rejected by the advancing solidification front, which in turn may result in macrosegregation. ${ }^{18}$ ) Moreover, the the flow field in rimming ingots may also play a major role in determining the grain structure of the finished product. While there is a good qualitative appreciation of the flow field generated by rising bubbles in rimming ingots, no quantitative description of the flow field has been attempted up to the present.

Let us consider fluid motion in a rectangular ingot, caused by rising gas bubbles, as sketched in Fig. 19. 


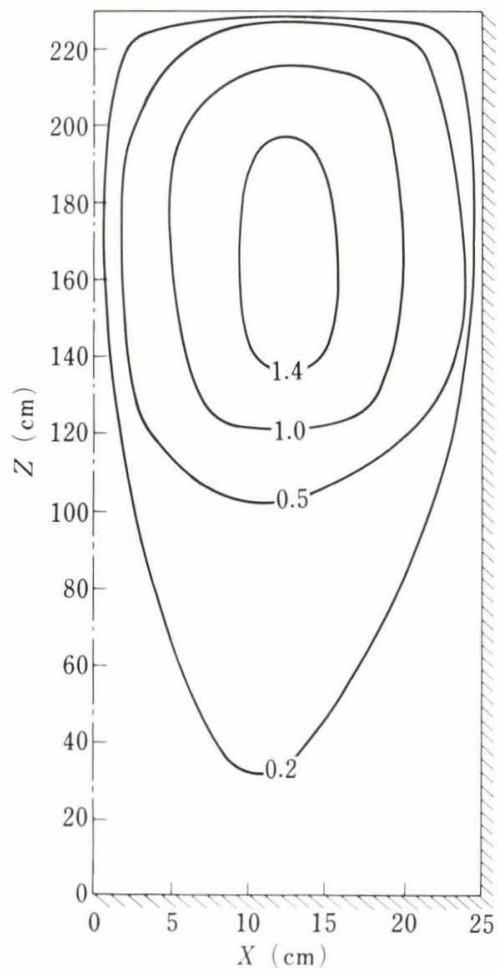

Fig. 23. Computed streamline pattern for a rimming ingot where gas evolution takes place only in the upper half of the vertical wall

In developing the mathematical statement of the problem the "K-W Model " was used for representing the turbulent viscosity, while the boundary conditions for the free surface, the bottom wall and in the central plane, were quite similar to those discussed in the previous examples. In the present case the driving force for the circulation is provided by the gas bubbles rising along the side walls, and the boundary conditions pertaining to the side walls constitute the major difference.

In the present case, as first approximation, a constant vorticity was assumed for the side wall, such that the velocity in the vicinity of the vertical walls was approximately $30 \mathrm{~cm} / \mathrm{sec}$; this velocity corresponds to the rising velocity of large spherical cap bubbles. A selection of the computed results is given in Figs. 20 to 23 which were computed for a vorticity at wall of $6(1 / \mathrm{sec})$ and for an ingot, $50 \times 230 \times(80) \mathrm{cm}$.

Figure 20 shows the computed streamlines pattern for a half-section which indicates a unicellular circulation pattern. The corresponding velocity field is shown in Fig. 21 where the velocity vector corresponding to $20 \mathrm{~cm} / \mathrm{sec}$ is also given for the sake of comparison. These results indicate quite rapid fluid motion in the vicinity of the walls and in the central plane, while the melt appears to be nearly stagnant in certain portions of the melt.

The map of the turbulent energy, shown in Fig. 22, indicates very high values, especially in the vicinity of the wall. These very high values of the turbulent energy would be consistent with the high fluid velocities calculated for systems with larger dimensions.

Finally, Fig. 23 shows the streamline pattern for a rimming ingot where the rimming action is assumed to be confined to the upper half of the vertical walls, which would correspond to weak rimming action. It is seen that under these conditions circulation is essentially confined to the upper part of the ingot while the lower regions appear to be largely stagnant.

It is to be stressed that these calculations for rimming ingots are very preliminary in nature; in particular, it would be desirable to refine the boundary conditions assumed for the vertical walls where the rimming action takes place. Perhaps such a more refined model may be developed once experimental data are available for the statement of more realistic boundary conditions.

\section{Concluding Remarks}

In the previous paper, ${ }^{1)}$ a mathematical representation has been proposed for a range of steel processing operations by using turbulent flow theory and a recently developed computational algorithm.

In this paper, the mathematical model is applied to the examples which include argon-stirred ladles, mixing in the pool of continuous casting systems, the flow field induced by a magnetic force field in continuous casting, and flow patterns in rimming ingots.

The computed results presented here were shown to be in good semi-quantitative, and in some instances quantitative, agreement with the results of experimental model tests for situations where such data were available.

While the computer time requirements are quite substantial, typically 5 to $10 \mathrm{~min}$ on the CDC 6400, the approach outlined here seems very attractive because it provides a much better insight into the structure of the flow field (including turbulence levels, the spatial distribution of the eddy diffusivity, etc.) than any simple physical modeling studies. This more detailed information on the flow field is thought to be an important starting point in further work aimed at the floatation and coalescence of inclusion particles, in the establishment of fluid flow effects on the morphology of solidified products, in the quantitative assessment of the effect of induction stirring and in a broad range of mass transfer operations involving liquid steel in turbulent motion.

A great deal of further work will be required before this computational technique will be immediately applicable to design; however, even the preliminary results which have been reported in this paper provide a much better insight into the behavior of these metals processing operations which should be immediately helpful-at least in a qualitative sense. It is hoped, moreover, that through the presentation of this work further experimental and theoretical studies will be stimulated.

\footnotetext{
Nomenclature

$C:$ concentration $(\%)$

$C_{i}$ : initial concentration of solute (\%)

$C_{m}$ : mean concentration (\%)

$C_{p}$ : specific heat $\left(\mathrm{cal} / \mathrm{g}{ }^{\circ} \mathrm{C}\right)$

$E$ : effective diffusivity $\left(\mathrm{cm}^{2} / \mathrm{sec}\right)$

$f_{l}:$ liquid fraction $(-)$

$f_{s}:$ solid fraction $(-)$
} 


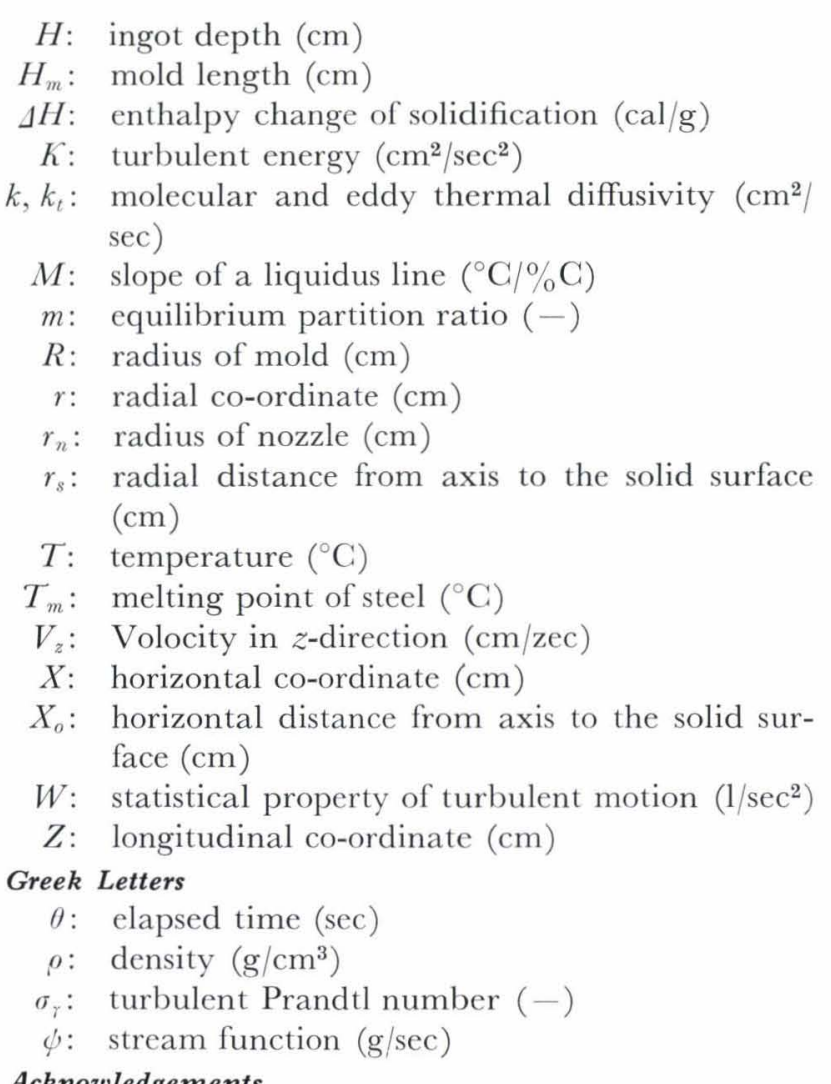

\section{Acknowledgements}

The authors wish to thank the A. E. Anderson Foundation for partial support of this investigation through a grant to the Center for Process Metallurgy, State University of New York at Buffalo.

\section{REFERENCES}

1) J. Szekely and S. Asai: Trans. ISIJ, 15 (1975), 270.

2) J. Szekely, S. Asai and C. W. Chang: Proceeding of the
International Iron and Steel Congress, Vol. III, Düsseldorf, May (1974), 5.2.2.1.

3) A. D. Gosman, W. M. Pun, A. K. Runchal, D. B. Spalding and M. Wolfshtein: Heat and Mass Transfer in Recirculating Flow, London and New York, Academic Press, (1969), 338.

4) K. Nakanishi, T. Fuji, H. Ooi, Y. Mihara and S. Iwaoka: Proceedings of the Fourth International Conference on Vacuum Metallurgy, Tokyo, (1973), 121.

5) W. M. Wojcik: Proceedings of the Continuous Casting Symposium of the 102nd AIME Annual Meeting, Chicago, 1973, 217.

6) P. H. Salmon and P. E. Hamill: Proceedings of the Continuous Casting Sympsoium of the 102nd AIME Annual Meeting, Chicago, (1973), 257.

7) Y Habu, H. Yoshii, H. Kitaoka, Y. Iida, T. Imai and T. Ueda: Tetsu-to-Hagané, 60 (1974), 962.

8) T. Kawawa, H. Sato, S. Miyahara, T. Koyano and H. Nemoto: Tetsu-to-Hagané, 60 (1974), 486.

9) M. Ishiguro, K. Kawakami, M. Ito and S. Miyoshi: Tetsuto-Hagané, 60 (1974), 885.

10) J. Szekely and R. T. Yadoya: Met. Trans., 3 (1973), 1279.

11) J. Szekely and R. T. Yadoya: Met. Trans., 4 (1973), 2673.

12) T. Mori, Y. Nagaoka, K. Orita and H. Sugitani: Tetsuto-Hagané, 59 (1973), A21.

13) S. Asai and J. Szekely: Ironmaking and Steelmaking (1975-in press)

14) K. Ushijima: Tetsu-to-Hagané, 48 (1962), 747.

15) E. A. Misikar: Trans. Met Soc. AIME, 239 (1967) 1747.

16) T. Fujii, J. Matsuno and H. Ooi: Tetsu-to-Hagané, 60 (1974), 1041.

17) A. A. Tzavaras: Proceedings of the Continuous Casting Symposium of the 102nd AIME Annual Meeting, Chicago, (1973), 197.

18) M. C. Flemings: Solidification Processing, McGrawHill, (1974)

19) J. K. Brimacombe and F. Weinberg: J. ISI, Jan., (1973), 24. 